University of Wollongong

Research Online

Faculty of Social Sciences - Papers (Archive) Faculty of Arts, Social Sciences \& Humanities

1998

The accessing of geometry schemas by high school students

Mohan Chinnappan

University of Wollongong, Mohan.Chinnappan@unisa.edu.au

Follow this and additional works at: https://ro.uow.edu.au/sspapers

Part of the Education Commons, and the Social and Behavioral Sciences Commons

Research Online is the open access institutional repository for the University of Wollongong. For further information contact the UOW Library: research-pubs@uow.edu.au 


\title{
The accessing of geometry schemas by high school students
}

\begin{abstract}
In this study I examine the question, what is the nature of prior mathematical knowledge that facilitates the construction of useful problem representations in the domain of geometry? The quality of prior knowledge is analysed in terms ofschemas that provide a measure of the degree of organisation of prior knowledge. Problem-solving performance and schema activation of a group of high- and low-achieving students were compared. As expected, the high achievers produced more correct answers than the low achievers. More significantly, schema comparison indicated that the high achievers accessed more problem-relevant schemas than the low achievers. In a related task which focused on the problem diagram, both groups accessed almost equal numbers of geometry schemas. The results are interpreted as suggesting that high achievers build schemas that are qualitatively more sophisticated than low achievers which in turn helps them construct representations that are conducive to understanding the structure of geometry problems.
\end{abstract}

\section{Keywords}

school, students, high, accessing, schemas, geometry

Disciplines

Education | Social and Behavioral Sciences

\section{Publication Details}

Chinnappan, M. (1998). The accessing of geometry schemas by high school students. Mathematics Education Research Journal, 10 (2), 27-45. 


\title{
The Accessing of Geometry Schemas by High School Students
}

\author{
Mohan Chinnappan \\ Department of Mathematics, University of Auckland
}

\begin{abstract}
In this study I examine the question, what is the nature of prior mathematical knowledge that facilitates the construction of useful problem representations in the domain of geometry? The quality of prior knowledge is analysed in terms of schemas that provide a measure of the degree of organisation of prior knowledge. Problem-solving performance and schema activation of a group of high- and lowachieving students were compared. As expected, the high achievers produced more correct answers than the low achievers. More significantly, schema comparison indicated that the high achievers accessed more problem-relevant schemas than the low achievers. In a related task which focused on the problem diagram, both groups accessed almost equal numbers of geometry schemas. The results are interpreted as suggesting that high achievers build schemas that are qualitatively more sophisticated than low achievers which in turn helps them construct representations that are conducive to understanding the structure of geometry problems.
\end{abstract}

The study of mathematical problem solving has received a great deal of attention for a considerable period. This is largely due to the fact that problem solving occupies a central role in mathematics learning and teaching and provides a window through which we can view how students grasp mathematical concepts and procedures. The primacy of problem-solving in mathematics teaching is reflected in major curriculum reform documents including Curriculum Evaluation and Standards (National Council of Teachers of Mathematics, 1989) where it is recommended that "problem solving should be the central focus of the mathematics curriculum" (p. 23). Accordingly, a considerable proportion of teachers' instruction time is directed at helping students become better problem solvers. Despite these efforts, students do not seem to perform as well as expected in tasks involving the solution of geometry problems (International Commission on Mathematical Instruction, 1995).

The current program of research into mathematical problem solving has examined many facets of mathematics problems and problem solving, including the skills and procedures involved in the solution process. More recently, however, one stream of research has focused on how students represent problems, and the function of representations in helping students arrive at or make progress towards solutions. Undoubtedly, this development constitutes an exciting area for problemsolving research, and it has the potential to provide an alternative perspective about why some students fail to solve problems and what we can do to help these students.

Representational studies of mathematical problem solving emerged from the desire to explain the nature of students' problem comprehension and the role that previously-learnt content knowledge plays during the construction of a particular representation. There is an emerging consensus that we need to investigate how 
the structure of prior mathematical knowledge influences students' problem representation and helps students become competent at solving problems. Consequently, the last decade has witnessed considerable investments in two fundamental aspects of mathematics problem representation: prior mathematical knowledge and use of that knowledge for problem representation. It is suggested that higher levels of problem-solving performance is dependent upon students' ability to develop a rich store of content knowledge and that patterns of use of the knowledge during problem representation could be influenced by the state of organisation of that knowledge (Prawat, 1989).

\section{Knowledge Organisation and Mathematical Activity}

In attempting to understand students' actions one could raise the question, what is the nature of prior mathematical knowledge that students bring to the task at hand? An understanding of this knowledge is important as it has implications for the progress made by the students with problems and activation of strategies that are relevant for their solutions.

There is a growing body of evidence to support the view that qualitative aspects of students' content knowledge could exert a major influence on the deployment of prior knowledge and the outcomes of students' problem-solving efforts. Quality of mathematical content knowledge could be interpreted in terms of the degree of organisation of the different bits of mathematical information. Network models of knowledge organisation (Rumelhart \& Ortony, 1977) provide a useful framework in which to visualise how mathematical knowledge could be organised. Well-organised knowledge can be seen as that which has many components built around one or more core ideas. There are connections between core ideas and their components, and among the components. The components could comprise mathematical definitions and rules as well as knowledge about how to deal with a particular class of problems. That is, organised mathematical knowledge encompasses both declarative and procedural knowledge (Anderson, 1995). Winne (1997) in his analysis of tactics for handling mathematics and other tasks, used the notion of "generic script" to refer to knowledge organised in this manner.

The issue of organisation of content knowledge in the human memory has led to the development of a key psychological framework called schemas. Schemas can be defined as clusters of knowledge that help students understand the problem and provide cues for the activation of relevant solution strategies. Marshall (1995) identified four primary components of schemas: recognition knowledge, constraint knowledge, planning knowledge, and implementation knowledge. She argued that the more tightly connected these components are, the easier it would be for the parts to be accessed and used flexibly. In a similar vein, Mayer (1992) has suggested that schematic and strategic knowledge are involved in any successful mathematical problem-solving effort, and that these knowledge forms have been neglected in studies of mathematics instruction and problem solving.

Studies of the performance of experts and novices have generated several hypotheses concerning the role of schemas and similar knowledge structures. In a study involving sorting of problems, Chi, Feltovich, and Glaser (1981) found that 
experts used schemas that were more often built around principles underlying the problems, compared to novices whose schemas tended to focus on superficial elements of problem statements and associated diagrams. The results led Chi et al. to conclude that this qualitative difference in schemas could explain why novices respond to the "surface structure" of a problem while experts respond to its "deep structure." Likewise, Chi, Glaser, and Rees (1982) ascribed expertise at problem solving to the structure of the domain knowledge base. They suggested that, in order to become an expert problem solver, one has to acquire a great deal of domain-specific declarative and procedural knowledge that are linked in meaningful ways.

Investigations conducted by Sweller $(1992,1994)$ and Cooper and Sweller (1987) in algebra led them to the conclusion that experts develop schemas that allow them to classify a given problem as belonging to a particular categorywhich in turn assists them in the retrieval of appropriate solution strategies. Owen and Sweller $(1985,1989)$ pursued the question of the importance of organising content knowledge in their study of trigonometry. The results showed that students who produced correct solutions in the least amount of time tended to access and use previously acquired schemas that were built around properties of right-angled triangles, including knowledge about how to deal with problems involving right-angled triangles. That is, students invoked schematised knowledge of trigonometry that was relevant to right-angled triangles. The investigators concluded that in order to become competent problem solvers, students must acquire an extensive body of domain-specific knowledge schemas. The development of domain-specific knowledge schemas of the type that Sweller and his colleagues were referring to could be characterised as involving the establishment of linkages between principles, rules, and concepts that solvers have to learn as they are introduced to a particular topic of mathematics.

The foregoing analysis suggests that successful students do utilise schema-like mathematical knowledge structures during problem solving. The study of how the disparate components of procedural and declarative mathematical information are linked, and the relationship between these structures and the outcome of a solution attempt, thus constitutes an important area of investigation. The study reported here takes up this question.

\section{Geometry Schemas}

While there is general agreement that schemas are knowledge structures which indicate chunking of information into meaningful wholes, authors differ in the way they specify the contents or components of schemas. As geometry schemas are the focus of this study, it is imperative that this term be defined more precisely here. The term schema is used in this study in two ways, the basic difference being one of magnitude and complexity. Firstly, on a macro level, a geometry schema can be visualised as a large chunk of information consisting of a core geometric concept; ideas about how to use that concept; and theorems, formulae and figures associated with that concept. For example, one can talk about a right-angled triangle schema (RATS). RATS could have several items of information linked to each other as shown in Figure 1. Of course this network of information could be 


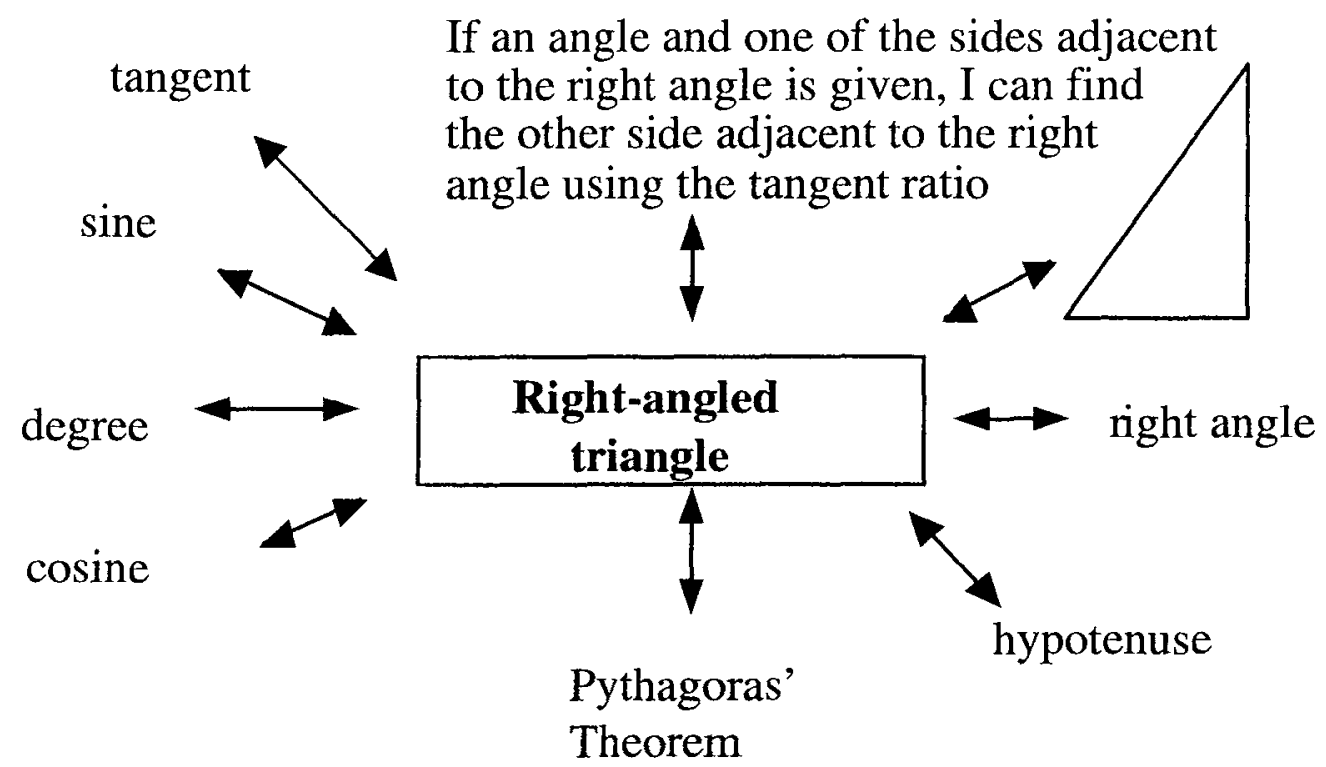

Figure 1. Information that is linked to form a right-angled triangle schema.

extended to include other information such as a proof of Pythagoras' theorem.

As students' knowledge of geometry increases, one would expect the RATS schema to become even more enlarged. For instance, the right-angled triangle could be rotated or it could be located within another figure such as a rectangle. Recognising a right-angled triangle within a square and activating related information can be seen as further development of students' RATS. Such a schema, which is rich in contents and multiple links, is considered to be sophisticated. Hence the quality of a schema can be interpreted in terms of the extent of the network of information built around a core geometric concept. The assumption here is that it is the more advanced or sophisticated schemas that play a key role in helping students understand and solve problems in a domain, a point highlighted by Nesher and Hershkovitz (1994).

On a micro level, the term schema could refer to clusters of information built around relatively smaller concepts. For example, the sine ratio is a schema in that there is a cluster of information built around it, including the meaning of terms such as opposite side, hypotenuse, and the ratio of two sides-not to mention the right-angled triangle and its transformations. In a sense, almost all basic concepts in geometry and trigonometry can be interpreted as schemas, the distinguishing feature being one of magnitude and the complexity of the links.

\section{Schema Activation and Problem Representation}

It is clear that performance in mathematical tasks is to a large measure dependent on using prior knowledge that is organised in the form of schemas. It seems that a major advantage of having knowledge stored in memory in clusters of schemas is that they facilitate retrieval of the required knowledge from the longterm memory into the working memory during cognitive processing. Let us now examine this advantage by turning our attention to the uses these schemas could be 
put to when students are engaged in mathematical tasks.

In problem-solving contexts, schemas could play an influential role during the construction of a representation for the problem. Cognitive psychologists argue that students' ability to solve mathematics problems can be greatly enhanced if they are taught to construct useful representations of the given problem (Frederiksen, 1984). Building a problem representation is a complex process in which students attempt to establish meaningful links between information in the problem statement and knowledge embedded in their schemas about that problem. The components of individual problem schemas could include (a) knowledge of procedures and strategies associated with tackling a group of problems that are similar to the problem in question, (b) mathematical concepts, and (c) knowledge about previous experiences with similar problems. Hence, building a representation of the problem involves constructing links between the above parts of the schema and information located in the problem. This point was highlighted by Hayes and Simon (1977) when they stated that "the representation of the problem must include the initial conditions of the problem, its goal, and the operators for reaching the goal from the initial state" (p. 21).

Thus, representation requires that connections be made between elements given in the problem and components that are present in the relevant schema accessed from memory. It follows that the more elaborate a schema is the greater the likelihood that students will be able to construct useful and multiple representations of the problem. It would appear that the richness of the problem schema plays a significant role in helping students to filter irrelevant information from given information and to attend to information that would be relevant to building representations.

A second area in which schematised mathematical knowledge can play a significant role in directing problem-solving processes is mapping, a strategy in which the solver attempts to establish a correspondence between the features and relations in a known problem (the base problem) with those of an unknown problem (the target problem). A successful mapping procedure requires that students go beyond the superficial aspects of the base problem in order extract its structure as encapsulated by key features and relations, and then use that structure to solve a new problem with a similar structure. English (1997) interpreted the former activity as constructing a meaningful mental model of the base problem. Information processing during mapping demands that students draw out the similarities between base and target problems. This is something experts would do more effectively and rapidly because their processing of problem structure is driven by more sophisticated and powerful schemas than those of novice problem solvers.

The function of schemas in the modeling process was investigated by Chinnappan (in press) in a study of problem-solving within the domain of geometry. The principal aim of the study was to examine the relationship between the quality of the schemas activated by students and the manner in which these schemas were deployed during the construction of mental models. The results of this study revealed that (a) students accessed a range of schemas relevant to the problem; (b) the successful students were able to align the schemas in ways that suggested an understanding of the problem structure; and (c) high-achieving 
students tended to build more complex mental models for the problem than low achievers, resulting in more novel paths to the solution. While the design of this study did not analyse transfer from a base to a target problem, the patterns of application of schemas suggested that students use knowledge structures acquired from previous attempts at solving problems in the general area of geometry. For example, students gain experience in solving unknown angles or sides of a rightangled triangle using knowledge about trigonometry. In doing so they acquire schemas that are built around right-angled triangles. Such schemas could consist of knowledge about properties of right-angled triangles, solution of equations, and use of trigonometric ratios. In a new problem that contains a right-angled triangle, students might have to use or generate information from the right-angled triangle in order to make progress towards the problem goal. However, the solution of a subproblem involving the right-angled triangle is unlikely to be achieved if the solver does not have a right-angled triangle schema or fails to activate one from long-term memory. In other words, the search in problem space involves the accessing and use of relevant schemas in order to generate new information which in turn could be used to solve other subproblems. The solution and management of these subproblems constitutes an important activity which, it can be argued, reflects the student's understanding of the structure underlying the problem in question.

The above interpretation of schema-driven problem search and mental modeling suggests that the study of schemas constitutes an important area in our understanding of the role of organisation of content knowledge in mathematical learning and problem solving. Specifically, the identification and probing of schemas that students activate in relation to the solution of a problem has the potential not only to provide insight into the type of schemas that students acquire in the general content area of a problem but also to reveal connections among those schemas that are required for the understanding of structural relations in a problem. Thus, the purpose of the present study was to generate data about the type of geometry schemas accessed by students and the utilisation of these schemas during the solution of a specific problem.

Two different but related strategies had to be used to generate data relevant to the question of the relationship between schemas and problem solving. In the first approach one could analyse schemas that are activated by students during the course of attempting to solve a problem. In this problem-solving context schema accessing can be argued to be controlled by the need to achieve a goal, i.e., the solution of the problem. Alternatively, one could design a task that is similar to the problem in that it has the basic structure of the problem but without the need to find an unknown. Completion of this task could involve students exploring it in ways that would allow them to activate schemas that might otherwise remain dormant. This latter approach provides a more open-ended approach that releases the student from the constraints of having to find an unknown value, thereby facilitating the accessing of a greater range of related schemas. It is assumed that a comparison of schemas activated during the above two contexts could provide us insight into the nature of schemas that were used by the students in understanding the structure of the problem.

The above logic led to the design of the present study in which I attempted to 
elicit schemas developed by students in the area of plane geometry in two contexts: problem and non-problem. Regardless of the context or the type of cue provided, it was predicted that students with high ability levels (high achievers) in mathematics would access more problem-relevant geometry schemas than their peers with low-ability levels (low achievers). It was further predicted that, in comparison to the low achievers, the activation of more problem-relevant schemas by the high achievers would be accompanied by superior solution outcomes.

\section{Method}

\section{Participants}

Thirty students from five Year 10 mathematics classes in a middle-class suburban high school volunteered to participate in the study. The school has a reputation for high academic standards and innovative teaching practices in the Brisbane metropolitan area. The mathematics classes at this school were ranked on the basis of students' mathematics performance in the previous years. Class rankings were relevant to the purposes of the present study because they provided a useful way to identify students with different levels of geometry knowledge schemas, the assumption being that students from the top-ranked class would have developed more extensive and sophisticated schemas that those from the lowerranked classes. The high-achieving group comprised 15 students from the topranked Year 10 class, while the 15 low achievers came from the bottom two Year 10 classes. Discussions with the teachers indicated that all students had completed the topic of geometry and trigonometry three weeks before the commencement of the study. In addition, most of the knowledge required for the solution of the target problem in the present study had been taught in the previous two years of the students' high school work.

\section{Tasks, Materials and Procedure}

The purpose of the present study necessitated strategies to gain access to schemas that students have developed in relation to the target problem. This was achieved by developing two tasks. The first task was a Plane Geometry Problem (PGP) which included a statement and a diagram (Figure 2). The problem consisted of three commonly encountered geometric forms: circle, tangent, and triangle. However, the problem was made more complex by having these forms integrated in a manner which demanded that the solver recognise a component as serving more than one function. For example, side AE needs to be identified as (a) a straight line, (b) a tangent to the circle, and (c) the hypotenuse of the right-angled triangle ACE. This recognition of one part of the figure as playing multiple roles constitutes an important prerequisite in the representation process before students could activate appropriate theorems and formulae. For instance, the recognition that $\mathrm{AE}$ is a tangent could help students infer that angle CDA is a right angle (radius-tangent theorem). Furthermore, the identification of segment $\mathrm{AE}$ as the hypotenuse of the right-angled triangle ACE could result in the use of Pythagoras' 
$\mathrm{AE}$ is a tangent to the circle, centre C. AC is perpendicular to $\mathrm{CE}_{\text {, }}$ and the angle DCE has a measure of $30^{\circ}$. The radius of the circle is equal to 5 $\mathrm{cm}$ Find $\mathrm{AB}$.

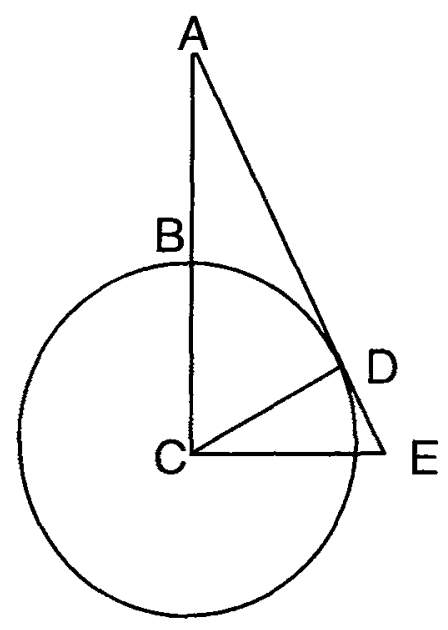

Figure 2. The Plane Geometry Problem (PGP).

OQ is perpendicular to OS. SQ touches the circle at $R$

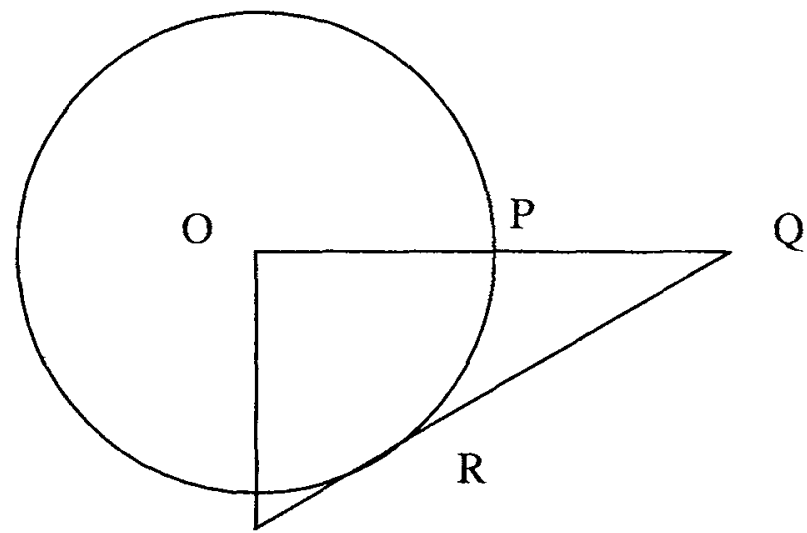

S

Figure 3. The Diagram Decomposition Task (DDT). 
formulae on the triangle ACE.

The second task of the study, the Diagram Decomposition Task (DDT) shown in Figure 3, was structurally similar to the PGP. However, it was different from the PGP in two aspects. Firstly, the DDT was not a problem-in the sense that it did not require students to solve for an unknown angle or side or prove any relations. As can be seen, the situation was essentially the same as the PGP except that any information about sides and angles was omitted. A second difference concerns the orientation of the figures: The DDT figure was obtained by rotating the PGP figure by 90 degrees. This change was introduced in order to encourage students to search for more schemas than were activated during completion of the PGP. The line joining the centre of the circle to the tangent was also removed in the DDT.

Participating students were interviewed twice. During the first session, students were given training in the use of think-aloud (Ericsson \& Simon, 1984) and diagram analysis techniques. During the second session, the PGP was presented on a $21 \mathrm{~cm} \times 13 \mathrm{~cm}$ card and students were encouraged to talk aloud as much as possible. After the first attempt, each student was prompted to try the problem again adopting different approaches. This procedure was expected to provide more opportunities for students to construct alternative representations of the PGP as well as yield more data about the range of geometry schemas built up by the students. The students' performance on the PGP was scored using a dichotomous scale. A score of 1 was awarded for one or more correct solutions and a score of 0 was awarded if students produced only incorrect solutions or failed to solve the problem.

Upon the completion of the problem-solving task, students were asked to work on the second task, the DDT. Firstly, students were asked to study the figure and then (a) identify all geometric forms that they could recognise; and (b) state any theorems, rules or formulae that they could associate with each of the forms they were able to recognise. Secondly, students were instructed to expand Figure 3 in any way they wished, after which they were asked to identify new forms and associated theorems that were created as a result of the additions they had made to the figure. All students' responses were video-recorded and transcribed.

Students might activate a variety of geometry schemas in the solution of the PGP, including schemas that may not be relevant to the problem. Because I was interested in examining students' schemas that were relevant to the PGP, it was necessary to develop a list of such schemas. In order to maximise data about problem-relevant schemas, I invited people with different levels of experience with the subject of geometry to attempt to solve the problem. The following participants were asked to solve the problem: a professional mathematician, two senior teachers of high school mathematics, and two high school students from another school. The participants' different experiences with geometry were also expected to provide a more complete range of schemas that one could associate with the problem in question. Analysis of the solution transcripts and responses to interview questions from these participants generated a total of 17 different problem-relevant schemas (Figure 4). Although one could not claim to have exhausted all the schemas that were necessary for the solution of the problem, the solution and interview responses did provide a degree of breadth and depth in the set of schemas. 


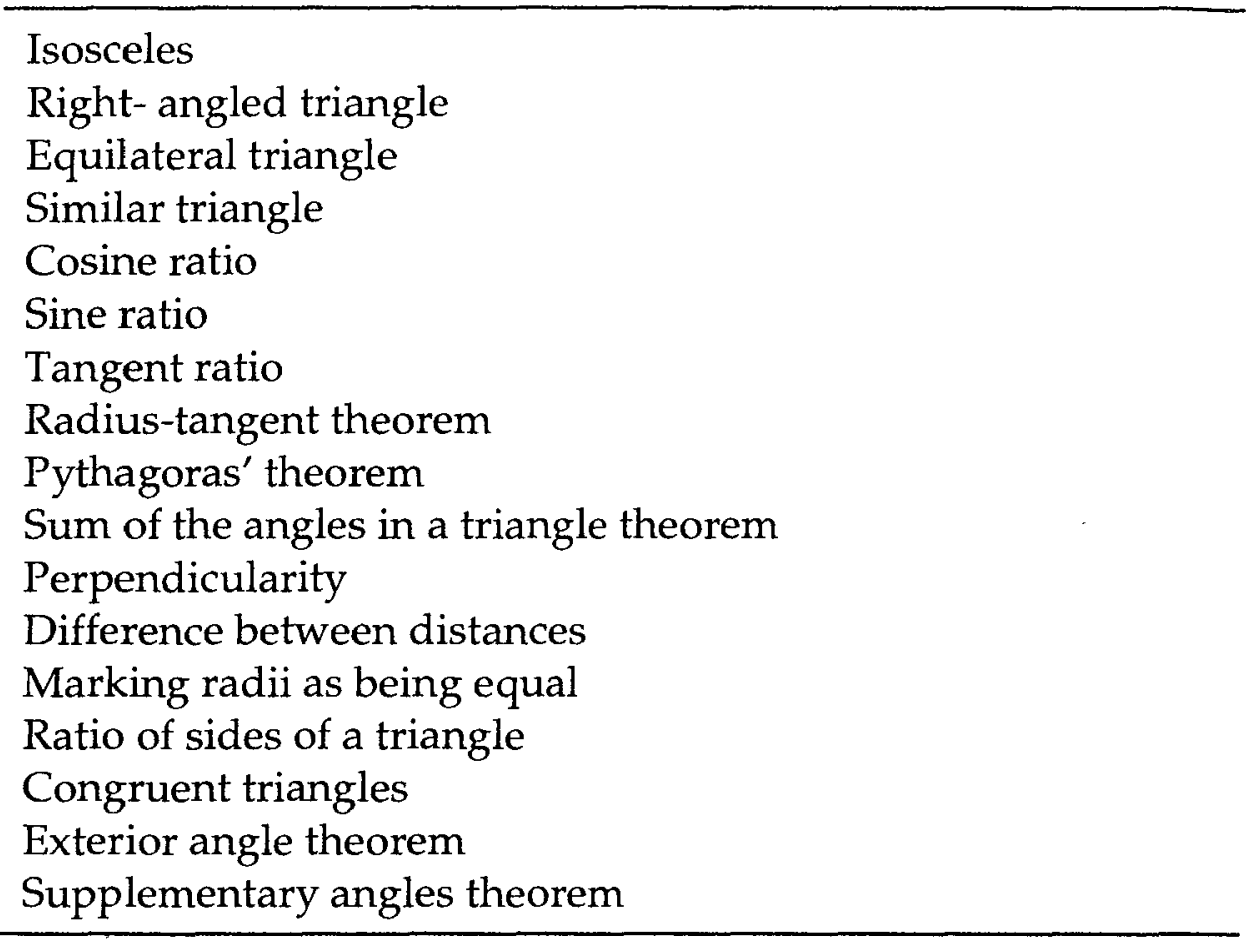

Figure 4. Geometry schemas necessary for solution of the PGP.

Unlike the problem-solving task, the DDT was open-ended; students could extend and explore the figure in any manner they wished as long as it was geometrically sound. As a consequence, the type and number of schemas students could activate was not so definite as for the PDP. In order get an approximate measure of the total number of different schemas that students could activate in expanding and analysing Figure 3, I searched their transcripts for schemas that were geometrically meaningful. A research assistant who is an experienced mathematics teacher was consulted in making decisions about the presence and correctness of the schemas. This exercise yielded a total of 70 different schemas (including those in Figure 4). Information about the remaining schemas is available from the author. Student extensions of the figure that were not geometrically sound were not included in the analysis. The frequency of activation of the different schemas was recorded.

\section{Results}

\section{Problem-solving Performance}

PDP solution scores for the two groups were compared using a $t$-test for independent samples. With an alpha level of 0.01 and a two-tailed test, the mean solution score for the high-achieving students $(M=0.73 ; S D=0.46)$ was significantly higher than the mean solution score for the low-achieving students $(M=0.07, S D=0.26), t(28)=4.91, p<0.001$. This result confirms that high achievers show superior performance in solving the target problem (the PGP) than low achievers. 


\section{Schema Activation}

The store of students' schematised knowledge of geometry was analysed by determining the frequency of the activation of these structures under two contexts: problem and non-problem. The results are given in Table 1.

In the problem context (the PGP), a frequency count was made of schemas that were used by high- and low-achieving students. As can be seen from Table 1, the high achievers accessed more than twice as many schemas as the low-achieving students in their problem-solving attempts.

The schemas activated in the non-problem context (the DDT) were analysed under four categories. The first category, called "Diagram Intact (open-ended)," consisted of the schemas accessed by students while they were analysing Figure 3. The category "Diagram Intact (problem-relevant)" consisted of the subset of these schemas that were relevant to a correct representation of the PGP. The third category, "Diagram Extended (open-ended)", consisted of geometry schemas that were activated as a consequence of expanding Figure 3. Finally, the "Diagram Extended (problem-relevant)" category consisted of the schemas from "Diagram Extended (open-ended)" that were relevant to the solution of the PGP. The frequency of each category among the two groups is given in Table 1.

Table 1

Numbers of Schemas Activated in Problem and Non-Problem Contexts, by Achievement Group

Context/category

Problem Context (PGP)

Solution of problem

Non-problem context (DDT)

Diagram Intact (open-ended)

Diagram Intact (problem-relevant)

Diagram Extended (open-ended)

Diagram Extended (problem-relevant)
52

Low achievers

High achievers

108

162

179

38

60

67

39

18

Table 1 shows that, although the high-achieving students activated a greater number of geometry schemas than their peers in the low-achieving group in the Diagram Intact (open-ended) category, the difference between the groups was marginal. Working within Figure 3 did not result in an appreciable difference in the number of schemas activated by the two groups of students. Contrary to expectation, when students were allowed to expand the figure, the low achievers tended to activate more schemas than the high achievers as shown by the Diagram Extended (open-ended) category. Taken together, the above patterns of schema activation suggest that both the groups had built up almost equivalent levels of geometry knowledge relevant to the DDT.

The pattern of results, however, is different when the analysis focuses on 
problem-relevant schemas. Knowledge relevant to the solution of the PDP is indicated by two categories: Diagram Intact (problem-relevant) and Diagram Extended (problem-relevant) in Table 1. The results show that high achievers activated a greater number of problem-relevant schemas than low achievers in the Diagram Intact situation. In the case of the Diagram Extended task, both groups accessed an equal number of problem-relevant schemas. The difference between the groups is more clearly shown by considering the number of problem-relevant schemas as a percentage of the total number of schemas activated. In the case of low achievers, $25 \%$ of the schemas were problem-relevant for the Diagram Intact task; the corresponding figure for the high achievers was $34 \%$. Comparison of the two proportions shows a significant difference $(z=-2.22, p<0.05)$. A similar pattern also emerges in the case of the Diagram Extended task, where the figures are $27 \%$ and $46 \%$ for the low and high achievers respectively. This difference in proportions of problem-relevant schemas is significant $(z=-12.6, p<0.01)$. Thus, in both situations the high-achieving students tended to build up and access proportionately more schemas that were relevant to the PGP than their peers in the low-achieving group.

Overall these results can be seen as providing evidence that both high-and low-achieving students develop reasonably extensive knowledge networks in the area of plane geometry, particularly around problems that are similar to the target problem of the present study. They also suggest that high achievers tend to ' construct more problem-relevant schemas than low achievers. That is, students who are seen as high achievers exhibit geometry schemas that are qualitatively superior to those shown by low achievers.

In order to highlight the above qualitative difference in the quality of schema activated by the two ability groups in the context of the DDT, I analysed and compared schemas produced by Ben (a low achiever) and Mike (a high achiever). Figure 5 shows Ben's expansion of the diagram and Figure 6 shows that of Mike. In both figures, lighter lines indicate the additions the students made to the original figure.

Ben added two lines (OU and $\mathrm{QU}$ ) to the given figure, resulting in a parallelogram OUQS. Having done this, Ben was able to generate several items of correct information about the parallelogram: angle SOU = angle SQU, angle OSQ = angle OUQ; OS = UQ. These bits of information form part of his schema about parallelograms. This information was classified as belonging to the category "Diagram Extended (open-ended)." While Ben's expansion of Figure 3 did use ideas that were part of his parallelogram schema, none of these was relevant to the understanding of the PGP. Not surprisingly, Ben did not solve the PGP. Ben was given a second attempt at the problem, but he again did not produce a correct solution. This suggests he did not have schemas that were relevant to a correct representation of the PGP, or at least that he did not access them.

Mike's analysis, shown in Figure 6, has a number of interesting features. He added three lines: TP, OR, and a line at point $P$ that ran parallel to OS. Mike then drew a number of correct geometric inferences from his modifications of the figure. Firstly, he recognised that OT was equal to OP, both being radii of the circle. This further led to him to recognise triangle OTP as isosceles. Secondly, the joining of points $\mathrm{O}$ and $\mathrm{R}$ helped him identify two right-angled triangles, ORS and ORQ. 
OQ is perpendicular to OS. SQ touches the circle at $R$

$\mathrm{U}$

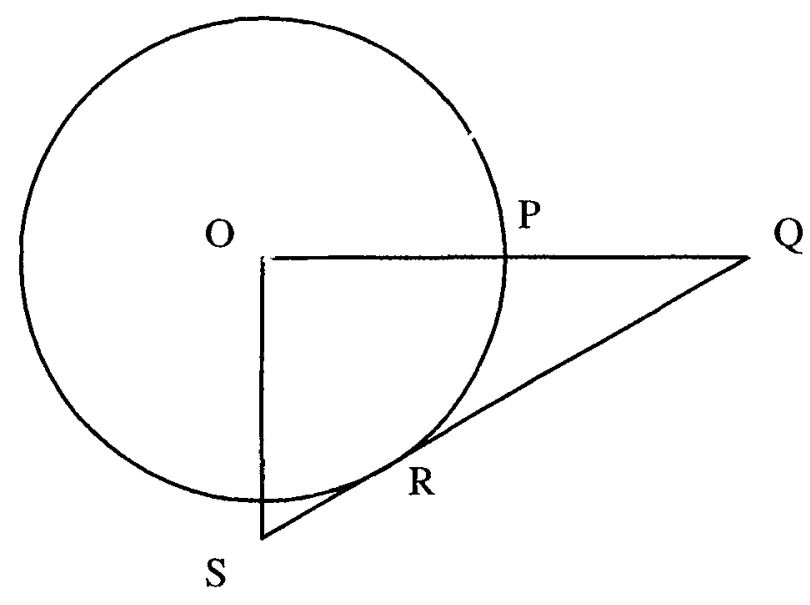

Figure 5. A low-achieving student's expansion of Figure 3.

OQ is perpendicular to OS. SQ touches the circle at $R$

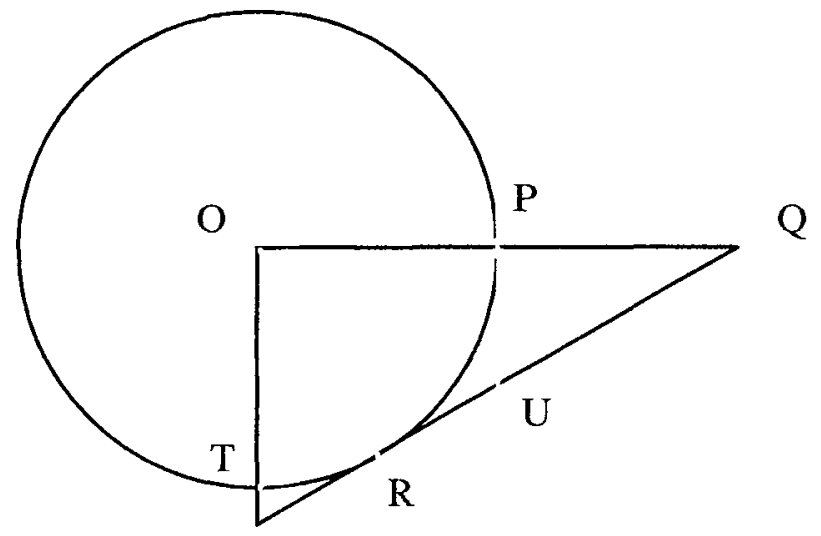

S

Figure 6. A high-achieving student's expansion of Figure 3. 
Mike was then able to access two items of relevant information that were connected to his schema about right-angled triangles: Pythagoras' theorem and trigonometric ratios. He went on to write equations that related the three sides of both the right-angled triangles by using Pythagoras' theorem. In addition, Mike showed that he could apply trigonometric ratios such as tangent, cosine and sine to work out angles and sides within the two right-angled triangles. Mike was also able to identify two right-angled triangles, QPU and QOS. A significant aspect of this part of his schema was that he recognised that these two triangles were similar. A fourth piece of information that Mike talked about arose from the line touching the circle at P. He inferred that because both the angles OPU and POS were right angles, the line PU was parallel to line OS. The first three pieces of information and their relations were crucial to discerning the structure of the PGP.

The range of schemas that Mike had built up about the given figure were not only more extended than Ben's, but more importantly they were instrumental in helping him represent and solve the PGP. This was reflected in the fact that not only was Mike able to solve the problem correctly at his first attempt, but that he was able to produce a correct alternative solution path during his second attempt. The second solution also showed that his range of schemas facilitate a flexible approach towards exploring different representations of the PGP. Here again, one could see evidence for the qualitatively superior schema of a high-achieving student.

\section{Discussion}

The aim of this study was to investigate problem-solving performance and the nature of schemas that students develop in the area of Euclidean geometry. Specifically, I sought to learn more about the quantity and quality of these domainspecific knowledge structures by examining schemas accessed by students in two contexts--problem and non-problem-and to explore the interrelations among these pieces of knowledge and students' understanding of the problem structure. Three hypotheses were central to the aims of the study. Firstly, it was predicted that high achievers would outperform the low achievers in producing the correct solution for the target problem. Secondly, it was hypothesised that high-achieving students would activate greater number of schemas than the low achievers during (a) the solution of the target problem, and (b) analysis of the diagram which reflected the structure of the target problem. Thirdly, I expected the high-achieving students to activate and use a larger number of problem-relevant schemas than low achievers during the completion of both tasks.

Comparison of the solution outcome scores for the two groups supported the first hypothesis that high achievers would do better than the low-achieving students. This was not surprising as one would expect the high-achieving students to be better problem solvers. However the question is, how can we explain their superior problem-solving performance? In the present study I have explored this question from the view point of quantity and quality of knowledge that students activate during the search for the solution and analysis of the problem diagram.

Frequency analysis of geometry schemas provided support for the prediction that students in the high-achieving group would activate a larger number of such knowledge forms than their peers in the low-achieving group during problem- 
solving situations. This suggests that the high achievers were more active than the low achievers in generating schemas that were potentially relevant to the production of the correct solution. While this was as expected, the data did not tell us much about the quality of the knowledge accessed by the high achievers in comparison to the low achievers. However, the superior solution outcomes produced by the high achievers suggests that most of the geometric schemas activated during the course of their solution process were indeed relevant to the solution. One could thus make judgements about the quality of the schemas by examining the relevancy of the schemas to the solution of the problem in question. I argue that schemas built by the high achievers were organisationally different from those of the low achievers, the assumption being that their better organised schemas allowed them to recognise the various relations embedded in the problem.

The greater incidence of problem-relevant schema activations by the high achievers was also evident in the second task (diagram analysis). While both the groups generated almost equivalent number of schemas, the high achievers activated a greater proportion of problem-relevant schemas than the low achievers. The microanalysis of the diagram expansions by Ben and Mike provides further support for the claim that high-achieving students had used more elaborate or sophisticated schemas than the low achievers in understanding the problem. Once again we have evidence that high achievers' schemas differ qualitatively from that of the low achievers.

When relevancy is not a consideration, ability level did not seem to have a significant effect on the quality of knowledge accessed. However, when the condition of problem relevancy was imposed, the high-achieving students tended to activate more schemas than the low achievers. A possible explanation for this difference is that schemas constructed by the high achievers were qualitatively superior. That is, these students were able to build multiple links between new geometric information presented to them and information that was already stored in their memory. For example, when a teacher discusses theorem that the diameter of a circle subtends an angle of ninety degrees at the circumference, students are generally given the figure or asked to deduce that the above theorem creates a right-angled triangle in a semicircle. Because the high achievers have already built up more conceptual points in their repertoire of mathematical knowledge, these students can now be expected to examine this information more critically and create more meaningful links than the low achievers. They could further invoke their prior knowledge about Pythagoras' theorem and trigonometric ratios and explore potential problems that could arise in a semicircle or they could link this theorem with other related theorems such as "angles subtended at the centre of the circle are twice those subtended at the circumference." As students build up these relations over a period of time, knowledge built around the core idea of right angles in a semicircle spreads in numerous meaningful directions. Anderson (1983) referred to this spread in knowledge networks as an important mechanism in building a large store of meaningful domain knowledge that supports problemsolving processes.

The more powerful and better-connected schemas exhibited by the high achievers play a vital role in facilitating the construction of representations of the given problem that reveal an understanding of its structure. In the present study, 
the high achievers were more adept at decoding the structure of the PGP as reflected by the greater number of correct solution outcomes produced by these students. Their schemas appear to be more powerful in "unpacking" the structure of the target problem. In contrast, schemas from the long-term memory of the low achievers were less sophisticated, and therefore, less effective in decomposing the problem in ways that would expose its structure. The lower degree of organisation of schemas developed by the low achievers was also reflected in these students activating fewer relevant schemas in conditions which demanded accessing and searching for solutions of the problem.

The results of this study are also consistent with findings of other studies that examined the relationship between the quality of mathematical knowledge and problem solving processes (Prawat, 1989; Shoenfeld, 1987). In his analysis of geometry problem solving, Shoenfeld showed that good students not only tend to build larger networks of mathematical knowledge than those who are not as good but, more importantly, their store of knowledge is better organised and more coherent. The better structured knowledge base of the high achievers of this study appeared to drive moves during their solution attempts. Newell (1990) drew attention to two types of searches in the problem space-problem search and knowledge search-both facilitated by a rich of store of schematised domain knowledge of the type built by the high achievers here.

High-achieving students' superior engagement with problem-relevant schemas can also be given an alternative explanation. While there is considerable support for the argument that successful problem solving in mathematics is based on extensive and well-organised knowledge in that domain, others have focused on general problem-solving skills and their role in the production of problem solutions (Lawson, 1991; Pressley, 1986). According to this line of thinking, effective solutions can be produced when students use general strategies in conjunction with their subject-matter knowledge. Students who invoke general strategies during problem solving are, it is argued, advantaged in two ways. Firstly, the use of these strategies tends to enhance the retrieval and manipulation of content-specific knowledge in the form of schemas that may otherwise have remained inert. Chinnappan and Lawson (1996) demonstrated that the use of certain search strategies could remind students to activate knowledge from memory. Secondly, general strategies help in the overall organisation and regulation of processes that are engaged in by the student (Glaser, 1984). These metacognitive actions inform the student about the progress made during the course of the solution process and help them assess the situation and look for alternative search paths, resulting in higher levels of knowledge generation and use. Hence, the net effect of applying general strategies is that high-achieving students adopt a planned and methodical approach in which geometric information is searched for more thoroughly and completely, and put to work more effectively, than is the case for low achievers.

The results of the present study also provide further support for the argument advanced by Alexander and Judy (1988) about the interaction between domain knowledge and domain-independent skills. While the results presented here did not examine domain-independent skills and the activation of knowledge of geometry, the marshalling of available schemas-particularly by students in the 
high-achieving group-suggest a possible interaction between the quality of geometric schemas and general skills that students could have acquired from the study of other mathematics topics. Chi (1985) suggests that when content knowledge is organised in a particular manner, the use of general strategies could be enhanced. In the present study the better connected geometry knowledge of the high achievers could have promoted the use of general strategies during the course of the solution process. In any event, the possible interaction between content knowledge and general skills is an important issue for mathematics educators which needs to be addressed in future research.

The range of schemas that both groups of students exhibited in this study indicates that the strategies adopted by their teachers appear to be working in helping students learn geometry in meaningful ways. The richness of schemas that students of both ability levels activated when they were required to expand the figure and identify associated theorems and formulae suggest that students' geometric knowledge is integrated to some degree. However, this level of integration appears to be insufficient when the task demands that schemas be utilised in uncovering the structure of the problem. Teaching of geometry has to explore ways of facilitating the construction of complex schemas. That is, we need to devise learning environments that have the potential to "help students develop knowledge structures which would make structural relations more salient" (Bassock, 1990, p. 532). Such schemas would facilitate the transfer of prior geometric knowledge to the representation and solution of novel problems.

In the present study I attempted to investigate the structure of geometric knowledge by examining the type of schemas students were able to activate in the context of a particular problem and its variant. While there is some evidence to support the claim that the quality of schematised geometric knowledge has a pivotal effect on representation of problems, the results are based on the finegrained analysis of one problem. There is, therefore, a need to replicate this study with a variety of two- and three-dimensional geometry problems and examine connections among the relevant schemas. Further, in making judgement about the quality of the schemas, I drew mainly on data about the relevancy of the schemas. Future studies need to generate more direct data about quality of schemas by examining the nature of relations that students build within and between schemas. The identification and specification of the links among geometry schemas, I would argue, is a critical cognitive issue as it has the potential to inform teachers about helping students, in particular the low achievers, understand problem structure.

\section{References}

Alexander, P., \& Judy, J. (1988). The interaction of domain-specific and strategic knowledge in academic performance. Review of Educational Research, 58, 375-404.

Anderson, J. R. (1983). The architecture of cognition. Cambridge, MA: Harvard University Press.

Anderson, J. R. (1995). Cognitive psychology and its implications (4th ed.). New York, NY: Freeman.

Bassock, M. (1990). Transfer of domain-specific problem-solving procedures. Journal of Experimental Psychology: Learning, Memory and Cognition, 16, 522-533.

Chi, M. T. H. (1985). Interactive roles of knowledge and strategies in the development of 
organised sorting and recall. In S. F. Chipman, J. W. Segal, \& R. Glaser (Eds.), Thinking and learning skills: Research and open questions (pp. 457-483). Hillsdale, NJ: Lawrence Erlbaum.

Chi, M. T. H., Feltovich, P. J., \& Glaser, R. (1981). Categorization and representation of physics problems by experts and novices. Cognitive Science, 5, 121-152.

Chi, M. T. H., Glaser, R., \& Rees, E. (1982). Expertise in problem solving. In R. J. Sternberg (Ed.), Advances in the psychology of human intelligence (Vol. 1, pp. 7-76). Hillsdale, NJ: Lawrence Erlbaum.

Chinnappan, M. (in press). Schema and mental models in geometry problem solving. Educational Studies in Mathematics.

Chinnappan, M., \& Lawson, M. (1996). The effects of training in use of executive strategies in geometry problem solving. Learning and Instruction, 6, 1-17.

Cooper, G., \& Sweller, J. (1987). Effects of schema acquisition and rule automation on mathematical problem-solving transfer. Journal of Educational Psychology, 79, 347-362.

English, L. (1997). The development of fifth-grade children's problem-posing abilities. Educational Studies in Mathematics, 34, 183-217.

Ericsson, K. A., \& Simon, H. A. (1984). Protocol analysis: Verbal reports as data. Cambridge, MA: MIT Press.

Frederiksen, N. (1984). Implications of cognitive theory for instruction in problem solving. Review of Educational Research, 54, 363-407.

Glaser, R. (1984). Education and thinking: The role of knowledge. American Psychologist, 39, 93-104.

Hayes, J. R., \& Simon, H. A. (1977). Psychological differences among problem isomorphs. In N. J. Castellan, D. B. Pisoni \& G. R. Potts (Eds.), Cognitive theory (Vol. 2, pp. 21-42). Hillsdale, NJ: Lawrence Erlbaum.

International Commission on Mathematical Instruction (1995). Perspectives on the teaching of geometry for the 21st century. Educational Studies in Mathematics, 28, 91-98.

Lawson, M. J. (1991). Testing for transfer following strategy training. In G. Evans (Ed.), Learning and teaching cognitive skills (pp. 208-228). Melbourne: Australian Council for Educational Research.

Marshall, S. P. (1995). Schemas in problem solving. New York, NY: Cambridge University Press.

Mayer, R. E. (1992). The psychology of mathematical problem solving. In F. K. Lester \& J. Garofalo (Eds.), Mathematical problem solving: Issues in research (pp. 1-13). Philadelphia, PA: The Franklin Institute Press.

National Council of Teachers of Mathematics (1989). Curriculum and Evaluation Standards for School Mathematics. Reston, VA: Author.

Newell, A. (1990) Unified theories of cognition. Cambridge, Mass: Harvard University Press.

Nesher, P., \& Hershkovitz, S. (1994). The role of schemes in two-step problems: Analysis and research findings. Educational Studies in Mathematics, 26, 1-23.

Owen, E., \& Sweller, J. (1985). What do students learn while solving mathematics problems? Journal of Educational Psychology, 77, 272-284.

Owen, E., \& Sweller, J. (1989). Should problem solving be used as a learning device in mathematics? Journal for Research in Mathematics Education, 20, 322-328.

Prawat, R. (1989). Promoting access to knowledge, strategy and disposition in students. Review of Educational Research, 59, 1-42.

Pressley, M. (1986). The relevance of the Good Strategy User model to the teaching of mathematics. Educational Psychologist, 21, 139-161.

Rumelhart, D., \& Ortony, A. (1977). The representation of knowledge in memory. In R. Anderson, R. Spiro \& W. Montague (Eds.), Schooling and the acquisition of knowledge (pp. 99-135). Hillsdale, NJ: Lawrence Erlbaum. 
Schoenfeld, A. H. (1987). On having and using geometric knowledge. In J. Hiebert (Ed.), Conceptual and procedural knowledge (pp 225-264). Hillsdale, NJ: Lawrence Erlbaum.

Sweller, J. (1992). Cognitive theories and their implications for mathematics instruction. In G. C. Leder (Ed.), Assessment and learning of mathematics (pp. 46-62). Melbourne: Australian Council for Educational Research.

Sweller, J. (1994). Cognitive load theory, learning difficulty, and instructional design. Learning and Instruction, 4, 295-312.

Winne, P. H. (1997). Experimenting to bootstrap self-regulated learning. Journal of Educational Psychology, 89, 397-410.

\section{Author}

Mohan Chinnappan, Department of Mathematics, The University of Auckland, Private Bag 92019, Auckland, New Zealand. Email: <chinnap@math.auckland.ac.nz>. 